\title{
Continuous Non-Invasive Blood Pressure Remote Monitoring System Microcontroller Based
}

\author{
Bharati S. Wakade ${ }^{1}$, Suresh A. Annadate ${ }^{2}$ \\ M.E. Student, Electronics Engineering Dept, M.G.M's Jawaharlal Nehru Engineering College, Aurangabad, India ${ }^{1}$ \\ Asst Professor, Electronics Engineering Dept, M.G.M's Jawaharlal Nehru Engineering College, Aurangabad, India ${ }^{2}$
}

\begin{abstract}
This paper presents design and development of a non-invasive method to measure the blood pressure signal in this instrument for remote monitoring and also continuous monitoring system based Zigbee module and microcontroller. The system is a embedded system and blood pressure is a biomedical signal measured using an optical device measurement circuit based photo plesthysmography technique continuously measure for a long period of time. Blood pressure numerical reading values of systolic and diastolic blood pressure calculated is then displayed on a mini LCD as well as stationary computer via Zigbee module and also obtained the results were compared with existing devices data as a Sphygmomanometer technique to verify the accuracy of the developed instrument.
\end{abstract}

Keywords: Blood Pressure, Zigbee module, mini LCD, PC, Photoplethysmography, Non-invasive.

\section{INTRODUCTION}

Blood pressure (BP) is the pressure exerted by circulating blood upon the walls of blood vessels and Blood pressure (BP) is measurement of the force applied on the walls of artery vessels as heart pumps blood through the body. Moreover, blood pressure measurement is known as one of the vital signs and is widely used to monitor the physiological condition of human beings along with other vital signs such as heart rate, breathing rate, oxygen saturation and temperature [1]. Blood pressure can be seen a two variance first one is a systolic Blood pressure (SBP) and second one is diastolic Blood pressure (DBP), and systolic is the higher amplitude pressure on the walls of the arteries which happens when the ventricles of the heart are contacting. While, diastolic is the lower amplitude pressure in the arteries, which happens near the end of the cardiac cycle when the ventricles are filled with blood and measured values for a healthy, resting adult or normal value are 115 to $120 \mathrm{mmHg}$ (millimeter of mercury) systolic and 75 to $80 \mathrm{mmHg}$ (millimeter of mercury) diastolic. Systolic and diastolic blood pressure measurements are not always constant and blood pressure does tend to change during the day. They are also change in response to stress nutrition, way of standard of living, illness drugs, and exercise [2].

The measurements of Blood Pressure are of great importance because it is used for detection of hypertension (high blood pressure). Blood pressure is low means the value is below $114 \mathrm{mmHg}$ is called as low blood pressure, and the value of the blood pressure is high means the value is above $130 \mathrm{mmHg}$ is called as the high blood pressure and the normal resting blood pressure for adult is approximately $120 / 80 \mathrm{mmHg}$. Hypertension is continues, consistent and independent risk factor for developing cardiovascular disease. Hypertension can cause the blood supply to the brain, heart and other tissues to be too low, and hypertension is strongly correlated with higher risk for cerebral stroke and heart infarct. Blood pressure measurement is also important for particular disease patients, and hemodialysis patients. Hence, in the daily life, blood pressure measurement and management is very useful for handling health situation and plays a preventive function.

Basically the blood pressure measure in two way Invasive blood pressure (IBP) and Non-invasive blood pressure (NIBP) in invasive measures the BP internally by using a sensitive IV catheter inserted into an superficial arty. Noninvasive method divided two way Auscultation/ Auscultatory (Manual Cuff) and Oscillometry[3]. These two non-invasive method are generally accepted and widely used but they severely restrain patient's mobility, they require uncomfortable cuffs; they are not suitable for home to measure the BP and cannot be used for continuous long-time monitoring applications. Continuous measurement of BP for homecare requires an accurate and inexpensive method that is independent form patient movement and does not require continuous care by a practitioner. These requirements can be found in this monitoring system which will be designed using photoelectric plesthysmography (PPG) technique [4].

\subsection{PPG Technique}

PPG means photoplethysmograhpic technique is noninvasive method by using this method to measure relative changes in pulse blood volume in the tissues. It utilizes the use of reflectance sensor that contains an infrared light source. The light source illuminates a part of the tissue (left hand fingertip) and a photo-detector receives the returning

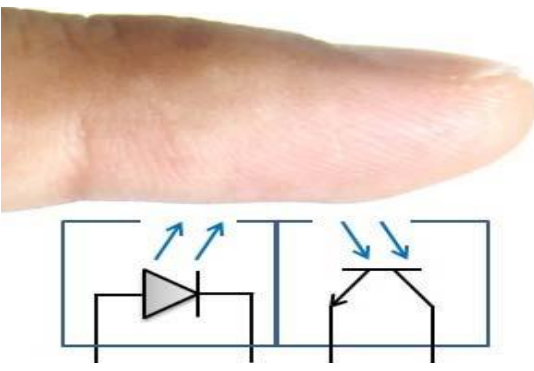

Fig. 1: Photoplethysmography technique 
INTERNATIONAL JOURNAL OF INNOVATIVE RESEARCH IN ELECTRICAL, ELECTRONICS, INSTRUMENTATION AND CONTROL ENGINEERING

light the waveform obtained from this technique represents the blood volume pulse which can be used to measure blood pressure [5].

\section{METHOD AND COMPONENTS}

The block diagram of the developed system is shown in fig. 2. The system mainly consists of three steps; the first is sensing measurement circuit second is signaling amplification circuit, third microcontroller circuit and transmission unit.

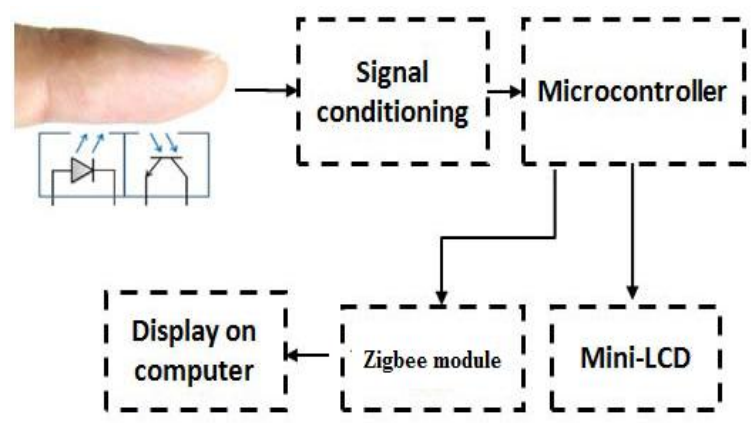

Fig. 2: Block diagram of our developed system

\section{A. Sensing stage}

In PPG technique detect the volume of blood pressure and pulse blood volume in a tissue in the arteries. The PPG basic form utilizes two components: source and detector. In source part a light source to illuminates a part of the tissue (e.g. left hand fingertip) and in detector part a photo detector to receive the light. Transparencies of living tissue to light make it possible for some part of the light form the source to pass through the tissue to the photo-detector. The pulse rate and blood pressure is inversely proportional to each other if the value of the blood pressure is increase the pulse rate value decrease and vice versa.

The volume of the blood in the vessel is variable while the volume of another part remains constant. Therefore the light absorption is varied only change blood volume increases or decreases and returning light to the photodetector changes according to the change of blood volume the changes electrical resistivity of the photo-detector depending on the amount of light falling on it. In this system optical sensor is used where it consists of infra-red emitting diode as the transmitter and a photodiode as the receiver.

\section{B. Signal conditioning stage}

The volume of the blood pressure signal is changes to detect that signal by using sensor, a low magnitude and low frequency biopotential signal is received by the photodiode. The sensor output signal is such a weak; it must undergo some signal conditioning means filtering and amplifying. So that it can be used for further processing. Since the photo-detector output voltage has a large amount of dc component which requires a filter to suppress out the dc component.

\section{Zigbee module}

This Zigbee RF4CE module is used in system the range is $75 \mathrm{~m}$ and the maximum data rate is $20-250 \mathrm{~KB} / \mathrm{s}$ and also used in another embedded system application.

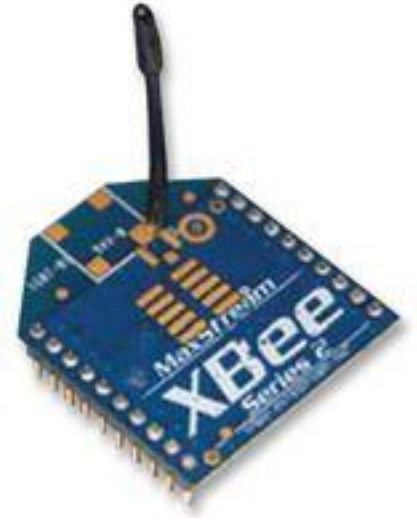

Fig. 3: Zigbee module

\section{Microcontroller stage}

The input of the microcontroller is output of the signal conditioning stage and the microcontroller processed (sampling and quantizing) the microcontroller $89 \mathrm{C} 51 / 52$ or $89 \mathrm{~S} 51 / 52$ is used in this system where it has a built-in an analog to digital convertor. The microcontroller identify the smallest value (DP) and largest value (SP) form the sensor output using a program written in kill software and the microcontroller sends the output signal, then displays the measured blood pressure readings in mini LCD and transmits them through a Zigbee module to any stationary enabled computer device.

The program flowchart is shown in fig. 4, first initialized the microcontroller and then set to the read the analog signal form the input. Timer interrupt is used here so that we can keep track if there are changes in the signal. The microcontroller then finds the largest peak of the signal and the lowest peak of the signal and then displays the systolic blood pressure and diastolic blood pressure readings respectively in the LCD and parallel serial terminal software via Zigbee module to the Computer.

By using the Zigbee module transmits the reading of SP and DP to the PC. The display on computer is acquired results through using special software called Parallel to Serial Terminal. It is intelligible terminal software which allows users to display predefined serial ports.

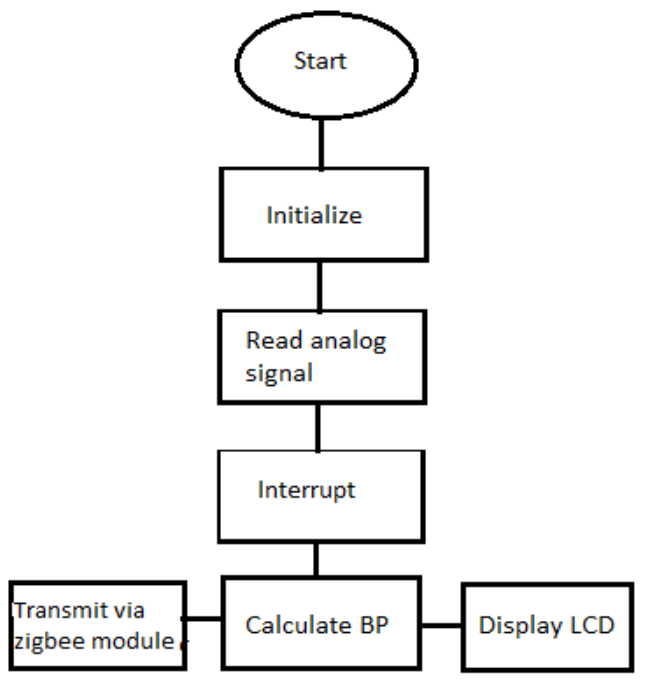

Fig. 4: Flow chart for the system 


\section{III.RESULT FOR THE SYSTEM}

The Fig. 5 shows the reading for Systolic pressure (SP) and diastolic pressure (DP) on mini LCD.

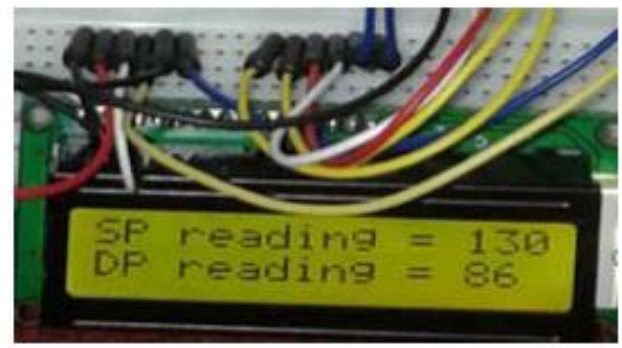

Fig. 5: Display the result of the developed system on LCD

The Fig. 6 shows that the microcontroller transmitted data by using the Zigbee module to the PC. Then also displayed this result on PC and store also.

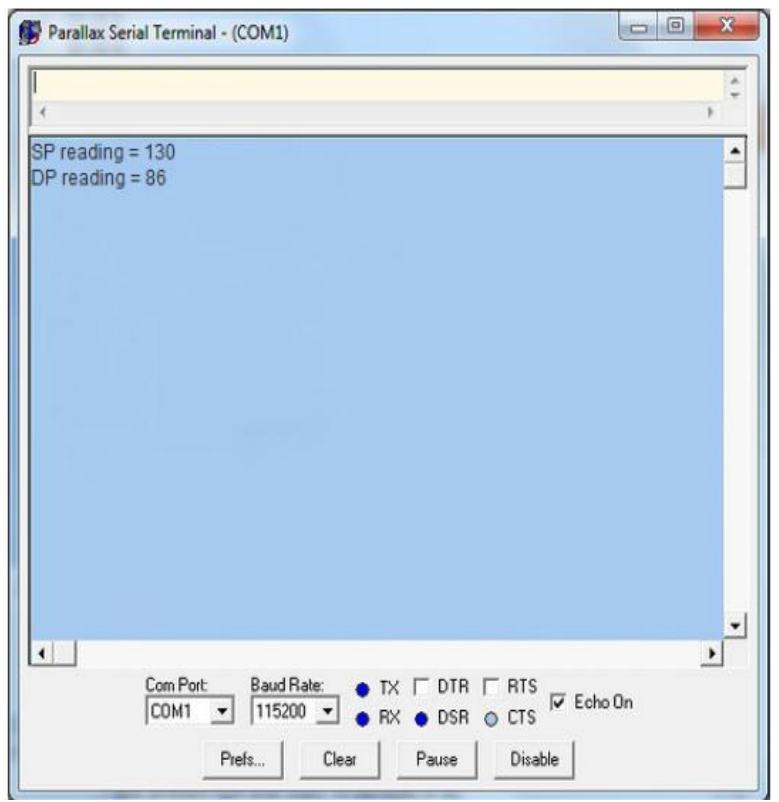

Fig. 6: Display the result on PC of developed system

\section{CONCLUSION}

In this paper, we developed Microcontroller Based Continuous Non-Invasive Blood Pressure Remote Monitoring System and the system based upon the Photoplethysmography technique. Continuously measured the blood pressure signal for a long period of time with the help of developed programming the small embedded system and displayed systolic and diastolic blood pressure in mini LCD and also transmits the systolic and diastolic value in PC via Zigbee module.

\section{REFERENCES}

[1] Jitha J Kollanoor, V. S. Suresh, "Remote Continuous Non-Invasive Blood Pressure Monitoring System With SMS Alert", International Journal of Engineering Sciences \& Emerging Technologies, Dec.2014. ISSN: 2231-6604, Volume 7, Issue 3, pp.687-692

[2] Basem Abu Zneid, Mohammed Al-zidi, Tareq Al-kharazi, "Noninvasive Blood Pressure Remote Monitoring Instrument Based Microcontroller", IEEE Region 10 Symposium, 2014 IEEE, Kaula Lumpur, Malaysia. DOI:10.1109/TENCOSpring.2014.6863036 pp. $248-253$
[3] Darovic, Gloriya Oblouk. Hemodynamic Monitoring: Invasive and Non-Invasive Clinical Application, $2^{\text {nd }}$ edition. Philadelphia: W.B. Saunders Company, 1995.

[4] Seppo N. , Milka S., Hannu S., Eija V. G., Risto M., NONINVASIVE BLOOD PRESSURE MEASUREMENT BASED ON THE ELECTRONIC PALPATION METHOD, University of Oulu, Department of Electrical Engineering, 1998.

[5] Md. M., Fida H., Rafi, Abu F., and M., A. Rashid, M. Fareq. Development of a Noninvasive Continuous Blood Pressure Measurement and Monitoring System. Department of Biomedical Engineering Khulna University of Engineering \& Technology. Khulna, Bangladesh. 2012l"'

\section{BIOGRAPHY}

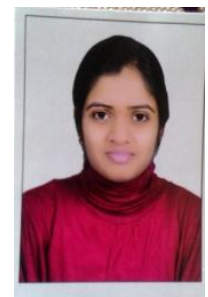

Bharati S. Wakade is pursuing M.E. in the stream of Electronics Engineering from M.G.M's Jawaharlal Nehru Engineering College Aurangabad, Maharashtra, India. She has completed her B.E. in the stream of Electronics and Telecommunication Engineering, from Savitribai Phule Women's Engineering College in 2014. Her areas of interest are Embedded Systems, Communication systems.

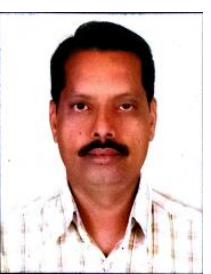

Suresh A. Annadate is working as Professor in the Department of Electronics Engineering at M.G.M's Jawaharlal Nehru Engineering College Aurangabad, Maharashtra, India. He has teaching experience of 23 years. He has published more than 50 papers in different national and international Journals. He has guided more than $100 \mathrm{UG}$ and PG students for their projects. His areas of interest are Embedded Systems, Communication systems, Digital Signal Processing and Signal Processing. 